\title{
Effects of $S$-adenosylmethionine on liver methionine metabolism and steatosis with ethanol-induced liver injury in rats
}

\author{
Zuojiong Gong $\cdot$ Shaonan Yan $\cdot$ Ping Zhang $\cdot$ \\ Yanqing Huang $\cdot$ Luwen Wang
}

Received: 5 November 2007/ Accepted: 8 May 2008/Published online: 25 July 2008

(C) Asian Pacific Association for the Study of the Liver 2008

\begin{abstract}
Background Hyperhomocysteinemia is implicated in the pathogenesis of various liver diseases. In this study, the effects of $S$-adenosylmethionine (SAM) on hyperhomocysteinemia and steatosis with ethanol-induced liver injury in rats were examined and their mechanisms were explored.

Methods Forty-eight female Sprague-Dawley rats were randomly divided into four groups as control, model, lowdose, and high-dose SAM groups. Except the control group, all rats were fed high-fat-containing diet plus ethanol and fish oil gavaged for 8 weeks. SAM was administered by intraperitoneal injection after the 4 weeks' exposure of ethanol. Serum homocysteine (Hcy), alanine aminotransferase (ALT), aspartate aminotransferase (AST), total cholesterol (TC), triglyceride (TG), tumor necrosis factor $\alpha$ (TNF- $\alpha$ ), and transforming growth factor $\beta 1$ (TGF- $\beta 1$ ) levels were determined. The contents of liver malondialdehyde (MDA) and glutathione (GSH) were assayed. Liver histology was also examined. The expressions of TNF- $\alpha$ and TGF- $\beta 1$ mRNAs in the liver were detected by the reverse transcriptase-polymerase chain reaction assay.

Results Compared with the control group, the model group rats developed marked liver damage, accompanied by an increase in Hcy, ALT, AST, TC, TG, TNF- $\alpha$, TGF$\beta 1$, and MDA levels. However, the levels of GSH were decreased. These responses were associated with the increased expression of TNF- $\alpha$ and TGF- $\beta 1$ mRNAs in the livers, as well as the existence of hepatocellular necrosis
\end{abstract}

Z. Gong $(\bowtie) \cdot$ S. Yan · P. Zhang · Y. Huang $\cdot$ L. Wang Department of Infectious Diseases, State Key Laboratory of Virology, Renmin Hospital of Wuhan University, Wuhan 430060, China

e-mail: zjgong@163.com and neutrophil infiltration in the livers. In treatment groups, SAM provided significant protection from the liver injury induced by alcohol, resulting in a decrease in serum TNF$\alpha$, TGF- $\beta 1$ levels, lipid peroxidation, and the expressions of TNF- $\alpha$ and TGF- $\beta 1$ mRNAs in the livers, as well as an increase in GSH levels. However, no statistical difference was observed in these parameters between the two different dose treatment groups. In the study, SAM did not affect plasma total homocysteine (tHcy) levels significantly.

Conclusion SAM prevents alcohol-induced liver injury in rats by reducing liver lipid peroxidation, anti-inflammation, and antihyperplasia. In addition, it does not affect the plasma tHcy levels.

Keywords Alcohol-induced liver injury .

$S$-Adenosylmethionine $\cdot$ Lipid peroxidation $\cdot$ TNF- $\alpha$. TGF- $\beta 1 \cdot$ Hyperhomocysteinemia

\section{Introduction}

Hyperhomocysteinemia is a common event in patients with liver diseases, especially in cases with alcoholic liver disease and liver failure. It has been implicated in the pathogenesis of these diseases. Increased homocysteine (Hcy) is one of the most important causes of endoplasmic reticulum stress. It promotes lipid synthesis and accumulation and induces apoptosis.

Previous studies have shown that chronic ethanol administration alters methionine in the liver, resulting in an increased intracellular $S$-adenosylhomocysteine $(\mathrm{SAH})$ and Hcy release into the plasma [1-3]. A major defect elicited by ethanol consumption appears to be the inhibition of methionine synthetase activity, which results in an impaired remethylation of Hcy to form methionine [4-6]. 
Ethanol consumption also increases the intracellular levels of SAH [7]. SAH is the metabolic precursor of Hcy and is formed as a product of methyl transfer reactions involving $S$-adenosylmethionine (SAM) [8-10]. Hcy is removed inefficiently during ethanol administration and with the impaired methionine synthetase activity. An increased accumulation of intracellular SAH eventually affects the activity of a number of methyltransferases. A special SAMdependent liver methyltransferase, phosphatidylethenolamine- $N$-methyl transferase (PEMT), which generates phosphatidylcholine (PC) from phosphatidylethanolamine via methylation, is especially susceptible [11]. It was reported recently that the generation of PC by the PEMT pathway is an obligatory event in the synthesis and secretion of very low-density lipoprotein [12]. This process is essential for lipid export from the liver. A defect in the PC generation via the PEMT pathway could, therefore, lead to abnormal lipid accumulation in the hepatocyte. Furthermore, proinflammatory cytokines such as tumor necrosis factor $\alpha(\mathrm{TNF}-\alpha)$ and transforming growth factor $\beta$ (TGF- $\beta$ ) play important roles in ethanol-induced liver injury. SAM is an essential metabolite in all cells and serves mainly as a methyl donor for many methylation reactions. It has even been used as a therapeutic agent in many toxin-induced liver injuries including those induced by alcohol [13]. The mechanism of their beneficial effects has not been clarified yet. The aim of this study was to investigate the efficacy of SAM in ameliorating ethanolinduced injury by improving methionine metabolism and steatosis in experimental rats.

\section{Materials and methods}

\section{Chemicals and reagents}

SAM (500 mg/amp) was kindly gifted by Knoll Pharmaceutical SPA Ltd. (Liscate, Italy). Ferrous sulfate was purchased from Shanghai Reagent Chemicals Co. Ltd (Shanghai, China). Hcy assay kit was purchased from Sigma-Aldrich Co. (USA). Enzyme-linked immunosorbent assay (ELISA) kits for rat TNF- $\alpha$ and TGF- $\beta$ detection were purchased from Shanghai Senxiong Biotech Industry Co. Ltd (Shanghai, China). Malondialdehyde (MDA) and glutathione (GSH) assay kits were purchased from Nanjing Jiancheng Bioengineering Co. Ltd (Nanjing, China). TRIzol reagent was purchased from Invitrogen Co. (USA). DL1000 DNA ladder marker was purchased from TaKaRa Biotech Co. Ltd (Japan). M-MLV reverse transcriptase, deoxyribonucleotide (dNTP, $10 \mathrm{mM}$ ), oligo (dT)15 primer, Taq DNA polymerase, and RNasin were purchased from Promega Biotech Co. Ltd (USA). Polymerase chain reaction (PCR) primers for TNF- $\alpha$, TGF- $\beta$, and GAPDH were synthesized by Sai-Bai-Sheng Biocompany (Shanghai, China).

Animal models

Forty-eight female Sprague-Dawley rats, weighing 100$150 \mathrm{~g}$, were obtained from the Experimental Animal Center of Wuhan University. After acclimation for 6-7 days, animals were randomly divided into four groups as control, model, low-dose, and high-dose SAM groups, with each group containing 12 rats. Except the control group, which was fed with ordinary diet and received an intragastric administration of physiological saline, other three groups were fed with ordinary foods with added fat-rich diet of lard and whole milk powder (80:10:10) and were administrated intragastrically with ethanol and $0.5 \mathrm{ml}$ fish oil. The initial dose of ethanol was $6 \mathrm{~g} \mathrm{~kg}^{-1}$ day $^{-1}$ (solutions maximally containing 56\% alcohol), and the dose was progressively increased during week 1 to a maintenance dose of $8 \mathrm{~g} \mathrm{~kg}^{-1}$ day $^{-1}$, which was continued for 8 weeks. SAM was administrated (100 and $\left.200 \mathrm{mg} \mathrm{kg}^{-1}\right)$ by intraperitoneal injection after exposure to ethanol for 4 weeks. Animals were weighed thrice per week. At the end of the experiment, animals were anesthetized with $20 \%$ urethane $\left(1.0 \mathrm{~g} \mathrm{~kg}^{-1}\right)$ and killed humanely by bleeding from femoral arteries. Blood and liver samples were collected for further examinations. All animals were given humane care in compliance with the institutional guidelines.

\section{Histological examination}

The liver specimens were fixed in $10 \%$ formaldehyde for $12 \mathrm{~h}$, embedded in paraffin, sliced into 5 - $\mu \mathrm{m}$ thick sections, and stained with hematoxylin-eosin. Histological assessments were performed blindly by two independent pathologists. The severity of steatosis was evaluated as 0 (no hepatocytes), 1 ( $<25 \%$ of hepatocytes), 2 (26-50\% of hepatocytes), 3 (51-75\% of hepatocytes), and 4 ( $>75 \%$ of hepatocytes). The necroinflammatory scores were evaluated as 0 (none), 1 (minimal), 2 (mild), 3 (moderate), and 4 (severe) on the basis of the degree of portal and lobular inflammation and the evidence of piecemeal and spotty necrosis.

Plasma total Hcy assay

Blood samples were collected in EDTA-containing tubes and centrifuged at 2,500 $\mathrm{g}$ for $10 \mathrm{~min}$, and the plasma was isolated and stored at $-20^{\circ} \mathrm{C}$. The plasma level of total Hcy (tHcy) was assessed using Waters 2475 high-performance liquid chromatograph according to the assay described by Mourvaki et al. [14]. 
Biochemistry and determination of serum TNF- $\alpha$ and TGF- $\beta 1$

Serum alanine aminotransferase (ALT), aspartate aminotransferase (AST), triglyceride (TG), and total cholesterol (TC) were determined by routine laboratory methods using a Hitachi Automatic Analyzer (Hitachi, Inc., Japan). Serum levels of $\mathrm{TNF}-\alpha$ and TGF- $\beta 1$ were measured using a commercial kit (Shanghai Senxiong Biotech Industry Co. Ltd, Shanghai, China) by sandwich ABC-ELISA according to the manufacturer's instructions.

\section{Determination of liver MDA and GSH}

Liver samples were thawed, weighed, and homogenized at a ratio of $1: 9(\mathrm{w} / \mathrm{v})$ in $0.9 \%$ saline solution. The homogenates were centrifuged at $3,000 \mathrm{rpm}$ for $10 \mathrm{~min}$ at $4{ }^{\circ} \mathrm{C}$. The supernatant was used for the determination of MDA, GSH, and total protein. MDA was assayed by spectrophotometric analysis for the levels of thiobarbituric acid-reactive substances at a wavelength of $532 \mathrm{~nm}$. The values were expressed in nmol $\mathrm{mg}^{-1}$ protein. Total GSH activities were also measured by spectrophotometric analysis. The absorbance was measured at $530 \mathrm{~nm}$. The activities of GSH were calculated and expressed as $\mathrm{U} \mathrm{mg}^{-1}$ protein. Total protein concentration was determined using the Coomassie Blue method, with bovine serum albumin as the standard.

\section{Detection of liver TNF- $\alpha$ and TGF- $\beta 1$ mRNAs}

Liver total RNAs were isolated by using the Catrimox-14 TM RNA purification kit (Takara Bio Inc, Japan). RNA concentrations were determined by spectrophotometric measurements at wavelengths of 260/280 nm. One microgram of RNA was transcribed into complementary DNA (cDNA) using AMV reverse transcriptase (Takara Bio Inc., Japan). The cDNA was amplified by PCR assay. The amplification primers for rat TNF- $\alpha$ were $5^{\prime}$-GCCAATGG CATGGATCTCAAAG- $3^{\prime}$ and $5^{\prime}$-CAGAGCAATGACTC CAAA- $3^{\prime}$, for rat TGF- $\beta 1$ were $5^{\prime}$-CACCATCCATGACA TGAACC-3' and 5'-TCATGTTGGACAACTGCTCC- $3^{\prime}$, and for rat GADPH were $5^{\prime}$-TCCCTCAAGATTGTCAGC AA- ${ }^{\prime}$ and 5'-AGATCCACAACGGATACATT-3', respectively. A $50 \mu \mathrm{l}$ of PCR reaction mix contained $10 \mathrm{mM}$ of dNTP, $2.5 \mathrm{mM}$ of $\mathrm{MgCl}_{2}, 20 \mathrm{mM}$ of Tris- $\mathrm{HCl}$ (pH 8.4), $50 \mathrm{mM}$ of $\mathrm{KCl}, 25 \mathrm{pM}$ of sense and antisense primers, and $2 \mathrm{U}$ of Taq DNA polymerase. Amplification was performed for 35 cycles with initial incubation at $94^{\circ} \mathrm{C}$ for $3 \mathrm{~min}$ and final extension at $72^{\circ} \mathrm{C}$ for $7 \mathrm{~min}$, with each cycle consisting of denaturation for $45 \mathrm{~s}$ at $94^{\circ} \mathrm{C}$, annealing for $45 \mathrm{~s}$ at $54^{\circ} \mathrm{C}$, and extension for $1 \mathrm{~min}$ at $72^{\circ} \mathrm{C}$. The PCR products were 357,404 , and 309 bp for TNF- $\alpha$, TGF- $\beta 1$, and GADPH, respectively. In all experiments, possible contamination with genomic DNA was excluded by PCR amplification in the absence of reverse transcriptase. The PCR products were electrophoresed on a $2 \%$ agarose gel. Semiquantitative evaluation was performed by using the Gel Doc 2000 System (BioRad Laboratories GmbH, Munchen, Germany). GADPH was used as a positive internal control and was found to be positive for each specimen. Its expression was used as a correction factor for TNF- $\alpha$ and TGF- $\beta 1$ mRNAs; thus, the results were calculated as the ratio of the intensity of bands of TNF- $\alpha$ and TGF- $\beta 1$ cDNA per GADPH cDNA on the gel.

Statistical analysis

All data were presented as means \pm SEM. Differences among groups were assessed by using unpaired Student $t$-test and one-way analysis of variance. $P$-values of less than 0.05 were considered to be statistically significant. Calculations were performed with the SPSS, Version 11.0, statistical software package.

\section{Results}

Changes in liver histology

The control group developed slight steatosis in the liver, but obvious inflammation or necrosis was not observed (Fig. 1a). In the model group, the rats developed hepatocyte steatosis, minimal to mild inflammation, and necrosis in the liver (Fig. 1b). However, both hepatocyte steatosis and inflammation were markedly reduced in the rats treated with low and high doses of SAM, except that only few fat vacuoles and inflammatory cells were observed (Fig. 1c, d). Liver steatosis scores and necroinflammatory scores in each group are referred in Table 1.

Effects of SAM on plasma tHcy, liver function, and blood fat levels

As shown in Table 2, the levels of plasma tHcy and serum ALT, AST, TG, and TC were significantly increased in the model group when compared with the control group. But there is no statistical difference in the levels of plasma tHcy between the model and the SAM treatment groups. Compared with the model groups, the levels of serum ALT, AST, TG, and TC significantly decreased in the SAMtreated rats.

Effects of SAM on TNF- $\alpha$, TGF- $\beta$, MDA, and GSH

As shown in Table 3, ethanol administration caused serum levels of TNF- $\alpha$ and TGF- $\beta 1$ to markedly increase in the 
Fig. 1 Histological

examinations of the liver: (a) normal group, (b) model group,

(c) low-dose SAM group, and

(d) high-dose SAM group.

Magnification, 200×
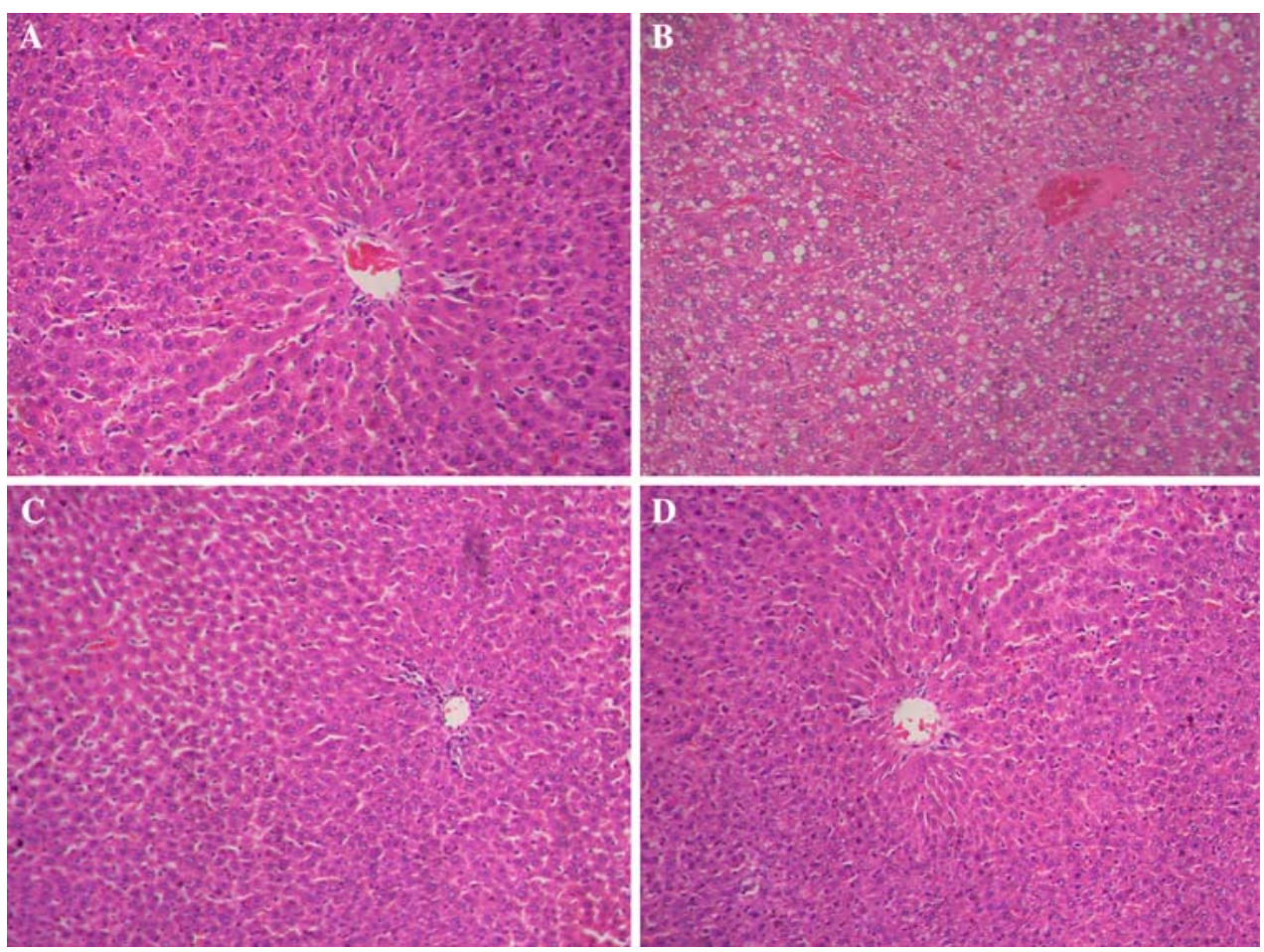

Table 1 Liver necroinflammatory scores and steatosis scores

\begin{tabular}{lllll}
\hline Groups & $n$ & Inflammation scores & Necrosis scores & Steatosis necrosis \\
\hline Normal control & 12 & $0.224 \pm 0.115^{\mathrm{a}}$ & $0.061 \pm 0.043^{\mathrm{a}}$ & $0.304 \pm 0.056^{\mathrm{a}}$ \\
Model & 12 & $2.963 \pm 0.393$ & $0.989 \pm 0.254$ & $3.372 \pm 0.237$ \\
Low-dose SAM & 12 & $0.331 \pm 0.123^{\mathrm{a}}$ & $0.074 \pm 0.026^{\mathrm{a}}$ & $0.351 \pm 0.032^{\mathrm{a}}$ \\
High-dose SAM & 12 & $0.301 \pm 0.227^{\mathrm{a}}$ & $0.069 \pm 0.135^{\mathrm{a}}$ & $0.346 \pm 0.106^{\mathrm{a}}$ \\
\hline
\end{tabular}

${ }^{a}$ Compared with model group, $P<0.01$

b Compared with model group, $P<0.05$

SAM, $S$-adenosylmethionine

Table 2 Detections of plasma tHcy, liver function, and blood fat levels

\begin{tabular}{lllcrrr}
\hline Groups & $n$ & tHcy $\left(\mathrm{mmol} \mathrm{l}^{-1}\right)$ & ALT $\left(\mathrm{U} 1^{-1}\right)$ & AST $\left(\mathrm{U}^{-1}\right)$ & TC $\left(\mathrm{mmol} \mathrm{1}^{-1}\right)$ & TG $\left(\mathrm{mmol}^{-1}\right)$ \\
\hline Normal control & 12 & $11.36 \pm 1.27^{\mathrm{a}}$ & $57.18 \pm 1.99^{\mathrm{a}}$ & $215.56 \pm 1.59^{\mathrm{a}}$ & $2.22 \pm 0.25^{\mathrm{a}}$ & $0.35 \pm 0.49^{\mathrm{a}}$ \\
Model & 12 & $24.64 \pm 2.47$ & $157.62 \pm 2.29$ & $247.78 \pm 1.93$ & $15.61 \pm 0.36$ & $2.4 \pm 0.18$ \\
Low-dose SAM & 12 & $23.30 \pm 3.34^{\mathrm{c}}$ & $64.35 \pm 1.62^{\mathrm{a}}$ & $189.39 \pm 6.45^{\mathrm{a}}$ & $3.53 \pm 0.29^{\mathrm{a}}$ & $0.57 \pm 0.20^{\mathrm{a}}$ \\
High-dose SAM & 12 & $22.45 \pm 2.66^{\mathrm{c}}$ & $63.75 \pm 2.85^{\mathrm{a}}$ & $187.50 \pm 5.54^{\mathrm{a}}$ & $3.49 \pm 0.32^{\mathrm{a}}$ & $0.54 \pm 0.31^{\mathrm{a}}$ \\
\hline
\end{tabular}

${ }^{\text {a }}$ Compared with model group, $P<0.01$

b Compared with model group, $P<0.05$

${ }^{c}$ Compared with model group, $P>0.05$

ALT, alanine aminotransferase; AST, aspartate aminotransferase; SAM, S-adenosylmethionine; tHyc, total homocysteine; TC, total cholesterol; TG, triglyceride

model group, but SAM administration could inhibit their increased levels after alcohol exposure. Compared with the normal group, liver MDA significantly increased in the rats of model group. However, SAM administration markedly blunted the increases of liver MDA. No difference existed in the contents of liver MDA between the SAM and the normal groups. Chronic fish oil plus ethanol gavage led to a marked reduction of GSH activity in the rats of model group, while SAM could significantly elevate the GSH activity. 
Table 3 Detection of TNF- $\alpha$, TGF- $\beta 1$, MDA, and GSH

\begin{tabular}{llllll}
\hline Groups & $n$ & TNF- $\alpha\left(\mathrm{pg} \mathrm{ml}^{-1}\right)$ & TGF- $\beta 1\left(\mathrm{pg} \mathrm{ml}^{-1}\right)$ & MDA $\left(\mathrm{nmol} \mathrm{mg}^{-1} \mathrm{protein}^{2}\right.$ & $\mathrm{GSH}\left(\mathrm{mg} \mathrm{mg}^{-1} \mathrm{protein}^{2}\right.$ \\
\hline Normal control & 12 & $3.84 \pm 0.12^{\mathrm{a}}$ & $20.45 \pm 1.36^{\mathrm{a}}$ & $2.82 \pm 0.16^{\mathrm{a}}$ & $26.87 \pm 2.33^{\mathrm{a}}$ \\
Model & 12 & $4.67 \pm 0.19$ & $26.39 \pm 1.21$ & $6.69 \pm 0.34$ & $11.38 \pm 1.98$ \\
Low-dose SAM & 12 & $4.19 \pm 0.40^{\mathrm{b}}$ & $25.09 \pm 0.83^{\mathrm{b}}$ & $1.65 \pm 0.51^{\mathrm{a}}$ & $19.50 \pm 1.77^{\mathrm{a}}$ \\
High-dose SAM & 12 & $4.05 \pm 0.58^{\mathrm{b}}$ & $24.46 \pm 0.50^{\mathrm{b}}$ & $1.63 \pm 0.17^{\mathrm{a}}$ & $20.47 \pm 0.85^{\mathrm{a}}$ \\
\hline
\end{tabular}

${ }^{a}$ Compared with model group, $P<0.01$

b Compared with model group, $P<0.05$

GSH, glutathione; MDA, malondialdehyde; SAM, $S$-adenosylmethionine; TGF- $\beta 1$, transforming growth factor $\beta 1$; TNF- $\alpha$, tumor necrosis factor $\alpha$

Effects of SAM on expressions of liver TNF- $\alpha$ and TGF- $\beta 1$ mRNAs

As shown in Fig. $2 \mathrm{a}, \mathrm{c}, \mathrm{TNF}-\alpha$ and TGF- $\beta 1$ mRNAs were barely detectable in the liver of control group rats, but after chronic ethanol gavages, the expressions of TNF- $\alpha$ and TGF- $\beta 1$ mRNAs were markedly induced. SAM administration could obviously inhibit the expressions of TNF- $\alpha$ and TGF- $\beta 1$ mRNAs in the rats of alcohol exposure group.

\section{Discussion}

At present, the most prevalent injury to the liver is induced by alcohol. However, its exact pathogenesis remains unclear. Hyperhomocysteinemia resulting from the disorder of methionine metabolism may play an important role in alcohol-induced liver injury [15]. The results of our study showed that the levels of plasma tHcy were significantly increased in the rats of model group compared with the control group. It is suggested that the disorders of methionine metabolism existed in alcohol-induced liver injury.

The results of our study also showed that the levels of plasma tHcy in both low-dose and high-dose SAM treatment groups could not be reduced compared with the model group. SAM could not improve hyperhomocysteinemia caused by alcohol-induced liver injury. However, the levels of serum ALT, AST, TG, and TC significantly decreased in the rats of SAM treatment groups when compared with the model groups. SAM could obviously improve liver injury induced by alcohol. The probable mechanisms were that SAM could decrease plasma levels of SAH, and thereby lead to an increase in the ratio of SAM and SAH, which could provide an active methyl donor for the normal methionine circulation for human body, eventually it could prevent the accumulation of SAH [16].

A lot of evidence showed that mediators of inflammation and cytokine play an important role in ethanol-induced liver injury, in which TNF- $\alpha$ may be the key factor $[17,18]$. The results of our study also confirmed that
Fig. 2 RT-PCR analysis of expressions of TNF- $\alpha$ mRNA and TGF- $\beta 1$ mRNA. (a) TNF- $\alpha$ mRNA, (b) GAPDH mRNA, (c) TGF-B1 mRNA, (d) GAPDH mRNA. Lane 1 represents normal group, lane 2 represents model group, lane 3 represents low-dose SAM group, and lane 4 represents high-dose SAM group. $\mathrm{M}$ represents molecular size marker
A TNF- $\alpha$ mRNA

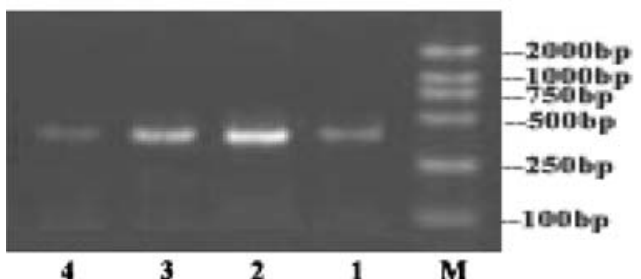

C TGF-B1 mRNA

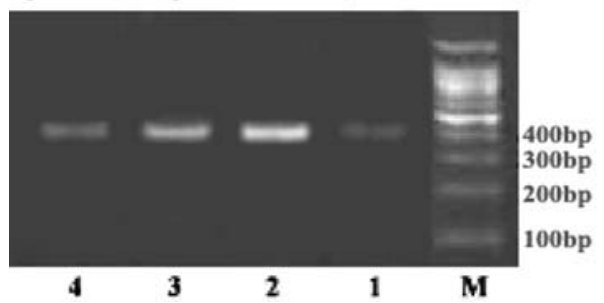

B GAPDH mRNA

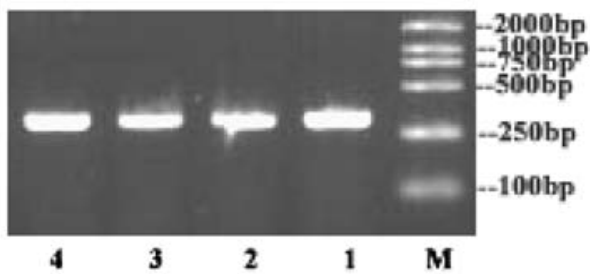

D GAPDH mRNA

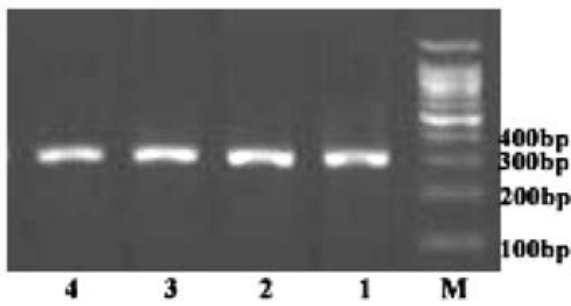


ethanol administration caused serum levels of $\mathrm{TNF}-\alpha$ to markedly increase in the rats of model group. We further observed that SAM administration could obviously inhibit the expressions of TNF- $\alpha$ mRNA and decrease the serum levels of TNF- $\alpha$ in the rats with alcohol exposure. It may be one of the reasons why SAM could protect liver injury induced by alcohol administration.

TGF- $\beta 1$ could induce the formation of extracellular matrix and inhibit the degradation of extracellular matrix, which could lead to the development of hepatic fibrosis. With frequent inflammation and fibrosis, alcoholic cirrhosis could finally emerge [19]. Therefore, downregulation of the expression of TGF- $\beta 1$ could delay the proceeding of hepatic fibrosis by preventing the apoptosis of hepatocyte and activation of hepatic stellate cells. The results of our study showed that ethanol administration caused serum levels of TGF- $\beta 1$ to markedly increase in the rats of model group, while SAM administration could obviously inhibit the expressions of TGF- $\beta 1$ mRNA, as well as decrease the serum levels of TGF- $\beta 1$ in the rats with alcoholic exposure. It is suggested that SAM had much effect on antihepatic fibrosis. The probable mechanisms could be that SAM blocked the production of collagen I by preventing TGF- $\beta 1$ to induce the COL1A2 promoter of collagen I [20]. Furthermore, 5'-methylthioadenosine, a product of SAM catabolism, could prevent upregulation of TGF- $\beta 1$ [21].

Alcohol and its metabolite, such as acetaldehyde and fatty acids, could generate a large amount of reactive oxygen species (ROS) in the course of metabolism by cytochrome p450 2E1 of microsomes. ROS could damage hepatocytes directly as well as by enhancing the sensitivity of hepatocytes to lipid peroxidation $[22,23]$. GSH is the important reducing agent in our body, which plays a key role against the toxic effects of ROS. Our study showed that liver MDA, a marker of lipid peroxidation, significantly increased in the rats of model group that had ethanol gavages for 8 weeks when compared with normal groups. However, SAM administration markedly blunted the rises of liver MDA. Furthermore, chronic fish oil plus ethanol gavages led to an obvious reduction of GSH activity in the rats of model group, while SAM could significantly elevate the GSH activity. Thus, SAM could increase the contents of GSH in liver microsomes and inhibit the production of MDA, which could enhance the ability for clearance of free radical and its metabolite, inhibit the reaction of lipid peroxidation, reduce the damages from free radicals, and protect hepatocytes.

In summary, the results of our studies indicated that SAM could prevent ethanol-induced liver injury in rats by modulating the system of oxidants and antioxidants in the liver, but did not affect the plasma levels of tHcy.
Acknowledgment This study was supported by the Scientific and Technology Bureau of Hubei Province Foundation (2007ABC010).

\section{References}

1. Bailey SM, Robinson G, Pinner A, Chamlee L, Ulasova E, Pompilius M, et al. S-Adenosylmethionine prevents chronic alcohol-induced mitochondrial dysfunction in the rat liver. Am J Physiol Gastrointest Liver Physiol 2006;291(5):G857-67.

2. Murillo-Fuentes ML, Artillo R, Ubeda N, Varela-Moreiras G, Murillo ML, Carreras O. Hepatic $S$-adenosylmethionine after maternal alcohol exposure on offspring rats. Addict Biol 2005;10(2):139-44.

3. Barak AJ, Beckenhauer HC, Mailliard ME, Kharbanda KK, Tuma DJ. Betaine lowers elevated $S$-adenosylhomocysteine levels in hepatocytes from ethanol-fed rats. J Nutr 2003;133(9): 2845-8.

4. Sakuta H, Suzuki T. Alcohol consumption and plasma homocysteine. Alcohol 2005;37(2):73-7.

5. Beulens JW, Sierksma A, Schaafsma G, Kok FJ, Struys EA, Jakobs C, et al. Kinetics of homocysteine metabolism after moderate alcohol consumption. Alcohol Clin Exp Res 2005; 29(5):739-45.

6. Barak AJ, Beckenhauer HC, Kharbanda KK, Tuma DJ. Chronic ethanol consumption increases homocysteine accumulation in hepatocytes. Alcohol 2001;25(2):77-81.

7. Kharbanda KK, Rogers DDII, Mailliarda ME, Sifordb GL, Baraka AJ, Beckenhauera HC, et al. Role of elevated $S$-adenosylhomocysteine in rat hepatocyte apoptosis: protection by betaine. Biochem Pharmacol 2005;70(12):1883-90.

8. Lin PY, Yang TH, Lin HG, Hu ML. Synergistic effects of $S$-adenosylhomocysteine and homocysteine on DNA damage in a murine microglial cell line. Clin Chim Acta 2007;379(1-2): 139-44.

9. Stam F, van Guldener C, ter Wee PM, Kulik W, Smithet DEC, Jakobs C, et al. Homocysteine clearance and methylation flux rates in health and end-stage renal disease: association with $S$-adenosylhomocysteine. Am J Physiol Renal Physiol 2004; 287(2):F215-23.

10. Poirier LA, Wise CK, Delongchamp RR, Sinha R. Blood determinations of $S$-adenosylmethionine, $S$-adenosylhomocysteine, and homocysteine: correlations with diet. Cancer Epidemiol Biomarkers Prev 2001;10(6):649-55.

11. Hendricks CL, Ross JR, Pichersky E, Noel JP, Zhou ZS. An enzyme-coupled colorimetric assay for $S$-adenosylmethioninedependent methyltransferases. Anal Biochem 2004;326(1):100-5.

12. Nishimaki-Mogami T, Yao Z, Fujimori K. Inhibition of phosphatidylcholine synthesis via the phosphatidylethanolamine methylation pathway impairs incorporation of bulk lipids into VLDL in cultured rat hepatocytes. J Lipid Res 2002;43(7):1035-45.

13. Aleynik S, Lieber CS. Role of $S$-adenosylmethionine in hyperhomocysteinemia and in the treatment of alcoholic liver disease. Nutrition 2000;16(11-12):1104-8.

14. Mourvaki E, Ferrante F, Ghirarduzzi A, Brini M, Depunzio I, Iorio A. Performance comparison of three assay methods used in fasting and postmethionine load plasma homocysteine determinations from patients with vascular disease. Am J Clin Pathol 2005;124(5):675-81.

15. Blasco C, Caballeria J, Deulofeu R, Lligona A, Pares A, Lluis $\mathrm{JM}$, et al. Prevalence and mechanisms of hyperhomocysteinemia in chronic alcoholics. Alcohol Clin Exp Res 2005;29(6):1044-8.

16. Kharbanda KK, Rogers DDII, Mailliard ME, Siford GL, Barak AJ, Beckenhauer HC, et al. A comparison of the effects of betaine 
and $S$-adenosylmethionine on ethanol-induced changes in methionine metabolism and steatosis in rat hepatocytes. J Nutr 2005;135(3):519-24.

17. Kishore R, Hill JR, McMullen MR, Frenkel J, Nagy LE. ERK1/2 and Egr-1 contribute to increased TNF-alpha production in rat Kupffer cells after chronic ethanol feeding. Am J Physiol Gastrointest Liver Physiol 2002;282(1):G6-15.

18. Song Z, Zhou Z, Uriarte S, Wang L, Kang YJ, Chen T, et al. $S$-Adenosylhomocysteine sensitizes to TNF-alpha hepatotoxicity in mice and liver cells: a possible etiological factor in alcoholic liver disease. Hepatology 2004;40(4):989-97.

19. Jiang W, Yang CQ, Liu WB, Wang YQ, He BM, Wang JY. Blockage of transforming growth factor beta-receptors prevents progression of pig serum-induced rat liver fibrosis. World $\mathbf{J}$ Gastroenterol 2004;10(11):1634-8.

20. Nieto N, Cederbaum AI. $S$-Adenosylmethionine blocks collagen I production by preventing transforming growth factor-beta induction of the COL1A2 promoter. J Biol Chem 2005;280(35): 30963-74.

21. Simile MM, Banni S, Angioni E, Carta G, De Miglio MR, Muroni MR, et al. 5'-Methylthioadenosine administration prevents lipid peroxidation and fibrogenesis induced in rat liver by carbon-tetrachloride intoxication. J Hepatol 2001;34(3):386-94.

22. Knerr S, Schaefer J, Both S, Mally A, Dekant W, Schrenk D. 2, 3, 7, 8-Tetrachlorodibenzo-p-dioxin induced cytochrome P450 s alter the formation of reactive oxygen species in liver cells. Mol Nutr Food Res 2006;50(4-5):378-84.

23. Parlesak A, Schafer C, Paulus SB, Hammes S, Diedrich JP, Bode C. Phagocytosis and production of reactive oxygen species by peripheral blood phagocytes in patients with different stages of alcohol-induced liver disease: effect of acute exposure to low ethanol concentrations. Alcohol Clin Exp Res 2003;27(3):503-8. 